\title{
MEMORIA, VERDAD, NACIÓN Y CIUDADANÍA: ALGUNAS REFLEXIONES SOBRE LA COMISIÓN DE LA VERDAD Y LA RECONCILIACIÓN EN SUdÁFricA ${ }^{1}$
}

\author{
Mónica Cejas
}

Resumen: En el presente articulo se yuxtapone "verdad" y memoria en el análisis del funcionamiento de la Comisión de la Verdad y la Reconciliación en Sudáfrica (1995-2001). Especialmente se exploran las "verdades" resultantes como producto de un proceso de redefinición de ciudadania y nación (un proyecto político de construcción-reconstrucción nacional) con cierta particularidad que conviene tenerpresente para poder pensarlas a la luz de similares experiencias en América Latina.

Palabras clave: Sudáfrica, memoria, verdad, ciudadanía, reconciliación.

Enviado a dictamen: 22 de febrero de 2007.

Aprobación: 09 de abril de 2007.

Doctora en Estudios Internacionales y Culturales por la Universidad Tsuda de Tokio (Japón), maestría en Estudios de Asia y África por El Colegio de México y en Estudios Internacionales y Culturales por la Universidad Tsuda, Tokio, Japón. Actualmente, es investigadora de la UAM-Xochimilco. Líneas de investigación: políticas de la memoria en Sudáfrica y Rwanda; género y feminismos en África; género y ciudadanía en África, correo electrónico: monicacejas@gmail.com.
Abstract: In the present article "truth" and memory are juxtaposed in the analysis of the South African Truth and Reconciliation Commission (TRC) (1995-2001). In the South African case, "truth" was the product of the process of new citizenship and nation-building. The South African experience is a way to rethink similar experiences in Latin America.

Palabras clave: South Africa, memory, truth, citizenship, reconciliation.

$\mathrm{E}$ 1 jurista peruano Carlos Chipoco señala que el derecho a la verdad tiene fundamentos doctrinarios jurídicos y prácticos; y que la verdad debe ser completa, oficial, pública e imparcial. La búsqueda de la verdad -en el caso de las Comisiones de la Verdad- es importante, dice, por un deber moral hacia las víctimas, los familiares y los deudos, para descubrir y sancionar a los culpables, para afirmar la democracia y el control ciudadano de las instituciones públicas y para evitar que las violaciones se repitan. Pero además, el respeto del derecho a la verdad es importante para abrir la posibilidad del perdón y la reconciliación, y para cumplir con el Derecho Internacional (Chipoco, 1994: 
83-106). Sin restar validez a estas afirmaciones de Chipoco, consideramos que se sitúan en el plano de lo ideal para una situación posttraumática como las dictaduras en América Latina y el apartheid en Sudáfrica. Porque si bien es cierto que las comisiones de la verdad son organismos de investigación creados para ayudar a las sociedades que han encarado graves situaciones de violencia política o guerra interna a enfrentarse críticamente con su pasado para superar las crisis y traumas generados por la violencia y evitar que se repitan en el futuro, sus condiciones operativas (quiénes las convocan, quiénes las conducen, su extensión, los poderes otorgados a los que están a cargo de las investigaciones y de la redacción del reporte final, los mecanismos para "llegar a la verdad", cómo y dónde o si se publican sus resultados, etcétera) determinan cómo se produce ese enfrentamiento crítico, cuáles son las verdades resultantes y su injerencia en el nuevo proyecto sociopolítico.

En este sentido hay que tener en cuenta que las comisiones de la verdad son el producto de negociaciones y como tales generan "verdades negociadas o de compromiso" que inscriben en el espacio público no sólo una lectura alternativa a la "verdad oficial" del régimen precedente sino una nueva "narrativa nacional". El trabajo de la comisión consiste no sólo en revelar esas verdades sino en convertirlas en una narrativa que partiendo de la memoria fragmentada, múltiple y muchas veces contradictoria de los eventos, institucionalice y fije, en cierto modo, una memoria del período en cuestión. En este sentido no puede negarse su carácter político en tanto dan voz a verdades no sólo silenciadas sino ideológicamente negadas y borradas del espacio público por el discurso oficial. Esto adquiere mayor significación si lo que se intentaron borrar son hechos de violenta negación de derechos (desapariciones, asesinatos, represión, etcétera). Se trata entonces de "verdades negociadas" que por lo tanto no pueden llegar a ser una verdad totalmente "completa e imparcial" como plantea Chipoco en tanto y en cuando no pueden reducirse a "una verdad unívoca" y se establecen en un tiempo frontera donde se yuxtaponen la narrativa aún presente del sistema que se trata de superar y las diversas propuestas (muchas veces en pugna) por el futuro alternativo, todo en el proceso de constitución de una comunidad política con una nueva identidad nacional basada en una nueva moral.

Sin la presencia de una conciencia política ampliamente difundida de que una amnesia colectiva sólo significa perpetuar la impunidad y el silencio y que por lo tanto el establecimiento de "la verdad" es un proceso necesario, las comisiones no pueden funcionar -esto explicaría el relativo fracaso de muchas de ellas-. En efecto, al momento de constituirse las comisiones, muchos de los personajes que animaron el antiguo régimen siguen ocupando puestos clave en estructuras militares, judiciales y políticas y más aún, los aparatos institucionales y todo el andamiaje legal de legitimación que las componían siguen contextualizando el espacio en que deben inscribir su accionar. Por otra parte, los sectores sometidos y violentados reclaman "justicia”, una moral sobre la cual pueda construirse un nuevo proyecto social cuya precondición es el reconocimiento de que esas verdades borradas en realidad fueron sumergidas y necesitan ponerse a flote, ser parte de la historia oficial en un acto público de reconocimiento. Las presiones que de ello surge condicionan muchas veces no sólo qué verdades se harán 
MEMORIA, VERDAD, NACIÓN Y CIUDADANÍA: ALGUNAS

públicas, sino la manera en que serán presentadas y el tipo de justicia que de ello se deriva. Cuando castigos concretos no pueden hacerse efectivos, puede optarse por los simbólicos debidamente ritualizados ya que resultaría quimérica una reconciliación sin justicia. $\mathrm{Y}$ es entonces que la amnistía, por ejemplo, como precondición para la "reconciliación", puede adquirir características especiales como veremos en el caso sudafricano.

Las verdades negociadas a que conducen las comisiones se gestan en tres instancias: durante las negociaciones que preceden la puesta en marcha de las mismas, durante su funcionamiento y al momento de decidir las formas y los contenidos de la narrativa que constituirá su reporte.

En este trabajo se propone analizar los significados, muchas veces tensos, de esas "verdades" y su proceso de constitución especialmente durante el proceso de audiencias y sin incluir el análisis del reporte por cuestiones de extensión.

\section{Contexto}

La redefinición de la ciudadanía en Sudáfrica comenzó con el discurso de F.W. de Klerk el 2 de febrero de 1990 donde se declaraba el fin del sistema de apartheid y dio su primer fruto el 27 de abril de 1994 con la realización de las primeras elecciones multirraciales en la historia del país. Esto puso en movimiento un nuevo proceso de construcción nacional.

La historia del apartheid puede interpretarse como la historia de los intentos por controlar el movimiento de la población mediante la creación de fronteras internas, la construcción de homelands y "estados independientes" y la correspondiente desnacionalización y renacionalización de la población doméstica con base en criterios étnicoraciales bajo un sistema que no sólo aseguraba el privilegio de unos pocos sino que también criminalizaba a los excluidos.

Durante el apartheid la premisa básica que regía los derechos correspondientes a la ciudadanía sudafricana era que "los derechos están investidos en la comunidad y que son conferidos al individuo por virtud de su pertenencia a esa comunidad" (Reitzes, 1995: 3). Esta ideología estaba legitimada por la Iglesia Reformada Holandesa (Dutch Reformed Church) que afirmaba que la separación entre razas era designio divino. En 1948, el Partido Nacional (National Party) combinó la segregación con la idea de supremacía de la raza blanca (baasskap) para garantizarse el monopolio del poder. En 1950 los nacionalistas afrikaners adoptaron el principio liberal de igualdad de libertades e intentaron combinarlo con el principio exclusivista del nacionalismo afrikaner. Entonces, argumentaban, la exclusividad social, política y cultural que demandaban para sí mismos podría ser garantizada a todos los pueblos (volkere) en Sudáfrica.

A mediados de los años sesenta los nacionalistas abandonaron la ideología basada en el concepto absoluto de baasskap y en la segregación como dominación y reformularon la ideología del apartheid en términos de "separados pero iguales". El país fue presentado no como nación-estado sino como un territorio acomodando muchas comunidades nacionales y abarcando varias naciones-estados. En 1971 se promulgó la Ley Bantu de Ciudadanía de los Homelands (Bantu Homelands Citizenship Act) completando el proceso de des-nacionalización de los africanos. En esta ley se definía sólo a los blancos como sudafricanos y a todos los africanos 
como ciudadanos de diferentes homelands. Los homelands no fueron creados por actos voluntarios de secesión de diferentes naciones para lograr la autodeterminación, sino que fueron creados por un grupo minoritario dominante que renacionalizó y dividió en multiplicidad de minorías a la mayoría de la población y la confinó a entidades territoriales separadas en las cuales resultaba imposible ejercer derechos económicos y en los cuales los derechos políticos no tenían significado. Entonces se crearon "Estados" sin soberanía real o autonomía dentro de un Estado. Los "otros" fueron construidos dentro del mismo Estado. El sistema de pases que regulaba los movimientos de población entre zonas urbanas y rurales fue abolido en 1986, pero hasta 1991, la definición oficial de inmigrantes establecía como condición esencial que se trate de una persona "asimilable" por su específica población blanca. La prolongada lucha contra el sistema generó también violencia y en algunos casos creó nuevas divisiones, odios y enfrentamientos (incluso entre africanos).

\section{La Comisión para la Verdad \\ y la reconciliación en Sudáfrica (TRC)}

Como señala Teresa Godwin Phelps (2004: 105), a diferencia de las comisiones de la verdad en América Latina cuyo principal objetivo fue reconstruir comunidades morales y políticas de lo que en teoría preexistía a los períodos de opresión, la de Sudáfrica fue pilar en la construcción de un Estado y una nación, agregaría, totalmente nuevos. La Constitución interina de 1993 fue el pacto de paz o compromiso político entre la minoría de las fuerzas del apartheid y la mayoría marginalizada y desprovista del derecho a voto liderada por el Congreso Nacional
Africano (ANC) que puso en marcha la llamada "transición". Se trataba de construir un puente entre el "pasado de una sociedad profundamente dividida, caracterizado por rivalidad, conflicto, innombrables sufrimientos e injusticia, y un futuro fundado en el reconocimiento de los derechos humanos, la democracia y la coexistencia pacífica y el desarrollo de oportunidades para todos los sudafricanos, sin consideraciones de color, raza, clase, creencia o sexo" (Constitution of the Republic of South Africa Act, 1993, sec. 232-4). Es decir, una nación que partiera de reconocer la diversidad, con un nuevo sentido de patriotismo, con igualdad de oportunidades que superasen las desigualdades políticas, económicas y de género del apartheid, con una cultura de democracia y derechos humanos. Este proyecto de transformación social así concebido tenía como textos subyacentes las ideas de reconciliación, reconstrucción y desarrollo como requisitos esenciales para la reconstrucción económica y moral del país. Y es como instrumento básico de este proyecto que debemos analizar a la Comisión de la Verdad. La voluntad política que impulsaba a la Comisión en el caso sudafricano, buscaba no sólo poner a flote las verdades sumergidas del apartheid, sino también el legado de violencia en la lucha antiapartheid liderada por el ANC. ${ }^{2}$ Crímenes del régimen y los perpetrados por aquellos implicados en una "guerra justa" contra el mismo. Se trataba de un proceso necesario para romper con el tabú de legitimidad moral de la lucha de liberación sometiéndola a investigación y de esta manera, al decantar sus delitos, reafirmar la justicia de la lucha. Todo en función de crear una memoria común reconocida por todos.

Una vez que la idea alcanzó cierto consenso se comenzó a reunir información sobre comisiones de la verdad en el mundo, se organizaron dos 
MEMORIA, VERDAD, NACIÓN Y CIUDADANÍA: ALGUNAS

REFLEXIONES SOBRE LA COMISIÓN DE LA VERDAD

Y LA RECONCILIACIÓN EN SUdÁFRICA

conferencias preparatorias con representantes de diferentes países y activa participación de organizaciones civiles.

La Comisión fue creada mediante una ley del Parlamento (the Promotion of Nacional Unity and Reconciliation Act, n.34 de 1995) a diferencia de los casos latinoamericanos donde fue establecida ya sea por iniciativa propia del ejecutivo (Decreto: Argentina y Chile) $)^{3}$, como resultado de Acuerdos de Paz con grupos opositores (El Salvador y Guatemala) ${ }^{4}$ patrocinados por Naciones Unidas o como una opción ética, un esfuerzo directo, a veces clandestino, de los activistas y organismos de derechos humanos como en Brasil, Uruguay y Paraguay. En África, de las ocho comisiones establecidas hasta la fecha, la de Sudáfrica fue la única creada por el Parlamento. Uganda, Chad y Nigeria fueron establecidas por decreto presidencial (1986, 1990 y 1999 respectivamente), Malawi por ley constitucional (1994), Sierra Leona por acuerdo de paz entre el gobierno y el Frente Unido Revolucionario (1999-2000), Burundi por iniciativa de Naciones Unidas (1995) y Zimbabwe por un grupo de activistas (1985). ${ }^{5}$

Piedra angular del nuevo proyecto de nación post-apartheid, la TRC ha mediado y enmarcado traumas individuales de manera de incorporarlos a una narrativa colectiva común instrumentalizada en nombre de la "nueva nación” (Grunebaum-Ralph, 2001:198). Este proyecto fue anticipado ya en la Constitución de transición -bajo un gobierno de unidad nacional- que desde 1993 a 1996 actuó como puente entre el marco legal que regía durante el apartheid y el que definitivamente regiría la Sudáfrica post-apartheid cuando se afirmaba la necesidad de "hacer memoria" para hacer frente a las divisiones y conflictos del pasado. En efecto, la idea de una Comisión de la Verdad en Sudáfrica surgió de los debates entre el gobernante Partido Nacional bajo el liderazgo del hasta entonces presidente, FW de Klerk, las fuerzas de seguridad lideradas por los generales y el ANC sobre las condiciones de la transición. El primero apoyado por sectores de la empresa y los medios, era partidario de una amnistía para los perpetradores del apartheid, mientras miembros del ANC eran partidarios de la realización de juicios criminales. La solución intermedia fue establecer una Comisión de la Verdad. La ley de Promoción de la Unidad Nacional y Reconciliación de 1995 confirió poderes a la Comisión de la Verdad para formular los medios de reparación y rehabilitación de dignidad civil y humana de las "víctimas" permitiéndoles testificar en sus propias palabras. También le daba autoridad para recomendar la amnistía a los "perpetradores" que confesasen de manera completa violaciones a los derechos humanos. ${ }^{6}$ Los primeros cinco volúmenes (2,739 páginas) con el reporte de los testimonios fueron entregados en octubre de 1998 al entonces presidente Nelson Mandela, en marzo de 2003 se entregaron dos volúmenes más al presidente Thabo Mbeki, cerrando oficialmente los trabajos de la Comisión.

$\mathrm{Su}$ mandato fue entonces ambicioso ${ }^{7}$ ya que tenía por objetivo promover la unidad nacional y la reconciliación en un espíritu de entendimiento que trascendiera los "conflictos y divisiones del pasado" mediante:

- El establecimiento de un panorama lo más completo posible de las causas, naturaleza y extensión de las graves violaciones a los derechos humanos cometidas entre marzo de 1960 (masacre de Sharpeville) y 1994 (por graves violaciones a los derechos humanos se entendía asesinato, secuestro, tortura y maltrato severo, 
es decir que se reducía a violaciones físicas);

- facilitar el otorgamiento de amnistía;

- $\quad$ establecer y hacer conocer el destino de las víctimas y restablecer la dignidad de las mismas y sus familiares mediante testimonio personal y recomendaciones para medidas de reparación;

- compilar un reporte incluyendo los resultados de las actividades mencionadas y con recomendaciones para prevenir futuras violaciones a los derechos humanos (Truth and Reconciliation Commission of South Africa Report 1 (4):54).

Para ello se le otorgaron poderes como el de allanamiento de propiedad y citación de testigos, también podía ofrecer un programa de protección de testigos. ${ }^{8}$

Respecto a los miembros de la comisión, en el caso sudafricano, se estableció una lista con trescientos nombres, muchos de ellos sugeridos por el público, que fueron sometidos a escrutinio por el gobierno y organizaciones civiles hasta quedar reducido a veinticinco personas. El presidente Nelson Mandela en consulta con su gabinete agregó quince más a la lista, finalmente se agregaron dos miembros más. ${ }^{9}$ Desmond Tutu, Arzobispo anglicano de Sudáfrica, fue nombrado presidente de la comisión y Alex Boraine, ministro metodista, ex diputado y fundador del Instituto por una Alternativa Democrática para Sudáfrica (IDASA), fue nombrado vicepresidente. La comisión contó con un presupuesto amplio (196 millones de rands $=310$ millones de pesos) $\mathrm{y}$ un staff de cuatrocientos investigadores $\mathrm{y}$ personal administrativo.

Para operacionalizar sus actividades la comisión se dividió en tres sub-comisiones: el Comité para las Violaciones de los Derechos Humanos, el Comité de Amnistía y el Comité de Reparación y
Rehabilitación. El proceso se dividió en tres fases: 1) algunas de las víctimas testificaron en audiencias públicas en diversos lugares del país, algunas de las cuales fueron transmitidas por radio y televisión (cuatro horas diarias de transmisión en vivo por radio, todos los días en el noticiero vespertino y los sábados en un especial de una hora por la emisora nacional de televisión SABC TV3, también en los periódicos) en los once idiomas oficiales; 2) luego se realizaron las llamadas "audiencias de amnistía" en las cuales los "perpetradores" (se usó el término en inglés perpetrators) testificaron y fueron sometidos a indagaciones para determinar si podían recibir amnistía; 3) finalmente, se realizaron audiencias con sectores específicos y a título corporativo: medios, empresas, religiosas, sindicatos, sector salud, prisiones, fuerzas armadas, etcétera. El proceso empezó, como ya se señaló, en 1995 y se concluyó con la entrega de los dos últimos volúmenes del reporte en marzo de 2003 (las actividades concernientes a audiencias públicas finalizaron en junio de 2001). ${ }^{10}$

Testificaron un total de 21 mil personas, de entre las cuales y después de un proceso de verificación se seleccionaron dos mil casos que fueron presentados en audiencias públicas. Las audiencias públicas comenzaron en abril de 1996 y se realizaron a lo largo de dos años; este comité terminó sus actividades en junio de 1998. El Comité de Amnistía recibió siete mil aplicaciones hasta 2001, de las cuales 5,287 fueron negadas y sólo aprobadas 568 . El comité de Reparación se encontró con la dificultad de que debido a la escasez de recursos resultaba casi imposible otorgar compensaciones individuales, por eso recomendó compensaciones simbólicas y reparaciones colectivas, incluyendo memoriales, monumentos y mejoras colectivas en educación o infraestructura. 
MEMORIA, VERDAD, NACIÓN Y CIUDADANÍA: ALGUNAS

REFLEXIONES SOBRE LA COMISIÓN DE LA VERDAD

Y LA RECONCILIACIÓN EN SUdÁFRICA

\section{Las verdades según la TRC}

La Comisión tuvo una relación ambivalente con el orden legal. No era una institución legal porque no se constituyó como una Corte y no determinaba la responsabilidad criminal ni podía dictar sanciones. Pero a su vez podía sobrepasar al sistema legal al clarificar una situación legal definida nombrando a los perpetradores antes de que fueran condenados y podía otorgarles amnistía antes de que fueran sometidos a juicio y condenados.

Aunque las Comisiones de la Verdad no pueden reemplazar al Poder Judicial ni al Ministerio Público y su finalidad no es presentar luego denuncias ante estas instancias, la TRC según Klopper (2001:456 y 461) tomó los procedimientos de la esfera legal y los transportó a la esfera pública haciendo uso de los conceptos jurídicos de evidencia e inferencia para arribar a la justicia. Como en las cortes judiciales, la evidencia legítima es aquella verificable, no contradictoria y coherente en los hechos. Sólo de este tipo de evidencia puede inferirse para establecer la verdad de los eventos. Pero las dificultades en mantener este discurso legalista quedaron de manifiesto cuando las nociones de justicia fueron reemplazadas por las de cura, cuidado de las víctimas y perpetradores, dando lugar a un discurso más psicológico que legalista que subrayaba las funciones terapéuticas de la comisión para llegar a "la verdad" mediante la revelación catártica de la misma exorcizando el dolor. Fundada en una aproximación centrada en las víctimas, la misma comisión que en uno de sus cuerpos podía conceder amnistías individuales, debía mantener un delicado balance que asegurase el reconocimiento de las víctimas como tales. El oprobio y la sanción pública de los perpetradores debían garantizar un mínimo de dignidad para las víctimas aunque el precio fuese una amnistía. Para poder orquestar esta catarsis, las audiencias crearon un espectáculo monumental en el ámbito nacional, enmarcadas en un ritual solemne, quasi-religioso y como acontecimiento político de nuevo tipo. Como veremos, el tipo de verdad resultante es más emocional que legal, una experiencia subjetiva más que un hecho objetivo. Todo cubierto por un discurso semi-religioso lo que no resulta sorprendente ante la presencia activa de representantes del clero cristiano en la comisión.

En el caso sudafricano la cuestión de "tratar con el pasado" fue interpretada con base en una narrativa moral y teológica (cristiana en particular), frecuentemente se hizo alusión a la metáfora biologicista de las heridas del cuerpo del individuo para simbolizar a su vez al cuerpo de la colectividad de la nación, de esta manera se incorporaba la experiencia individual al colectivo nacional. ${ }^{11}$ El 16 de diciembre de 1995, día en que la TRC dio inicio a sus actividades, Tutu abrió el acto señalando: "Se supone que somos parte del proceso de cura de nuestra nación, de nuestro pueblo, todos nosotros, ya que todo sudafricano ha sido, de una manera u otra, traumatizado. Somos un pueblo herido... todos necesitamos curar" (1999: 87).

De allí que sus funciones adquiriesen un carácter también terapéutico (Sanders, 2002: 66), curar haciendo que la verdad sea contada para entonces perdonar y lograr la reconciliación.

La primera audiencia pública del Comité para la Violación de los Derechos Humanos sentó precedente para el ritual que caracterizaría al resto. Celebrada en el auditorio de East London contó con la presencia de dos mil personas. La audiencia comenzó con el canto de un himno religioso en alguna Xhosa (haciendo referencia 
al perdón de los pecados) mientras las víctimas iban ocupando sus lugares en el recinto, quedando de manera ritualizada separados del resto de los asistentes. Luego Tutu hizo una oración a manera de introducción solicitando el auxilio divino para las actividades de ese día. A continuación se leyeron en voz alta los nombres de los muertos y desaparecidos cuyos casos se tratarían ese día. Luego se encendió una gran vela con una cruz roja y los miembros de la comisión pasaron a saludar personalmente a las víctimas mientras la audiencia contemplaba de pie (Antjie Krog, 1998: 22-23). Así, en otras audiencias, la ceremonia podía iniciarse con una oración cristiana, musulmana o judía, se encendía la gran vela blanca simbolizando la verdad, y entonces los siete comisionados ubicados tras una mesa cubierta de un lienzo blanco se dirigían a saludar a las víctimas. Cada víctima prestaba testimonio acompañada por miembros de su familia y un psicoterapeuta. Para comenzar, uno de los comisionados preguntaba el grado de parentesco del declarante con la víctima, su edad, domicilio, empleo, etcétera. Esto era importante según en el sentido africano de ubicar a la víctima en una familia, una comunidad, un lugar (VanZanten Gallagher, 1998). Al finalizar su testimonio, también se les preguntaba qué reparaciones deseaban; en todo momento, los comisionados subrayaban su papel de representantes del estado como garante de justicia. La audiencia era entonces un espectáculo que permitía hacer público el dolor, lograr el reconocimiento de la comunidad y trazar un mapa de los hechos.

Para el caso de la Comisión encargada de las amnistías, cabe señalar que no se trató de una amnistía concedida sin condiciones, sino de una amnistía específica decidida por un panel en una audiencia pública. El perpetrador debía inscribirse personalmente y en audiencia pública revelar por completo sus crímenes y demostrar la manera en que los había llevado a cabo. Todo ello en presencia de las víctimas y sus representantes, quienes podían participar en su interrogatorio. Debía tratarse de crímenes motivados por la defensa institucional o ideológica del régimen, lo que se llamó “crimen político", y no debería haber mostrado sadismo o interés personal en el mismo. La expresión pública de arrepentimiento no fue condición para otorgar la amnistía. En este caso el acto performativo completa en cierta forma el correspondiente a las audiencias para las víctimas porque permite que éstas se ubiquen del lado acusador, haciendo público no sólo lo acaecido a la víctima en las palabras del perpetrador, sino también, y con esto exponiéndolos, logrando la condena de la comunidad. Ya no son nombres en una lista sino rostros y voces perfectamente identificables. La transmisión por los medios contribuyó a multiplicar ese efecto, a tornarlo un evento nacional. Para los casos en que la amnistía era concedida, ésta significaba para los perpetradores una condena más "suave" que la que debería corresponderles de acuerdo con criterios de justicia retributiva.

Funciones jurídicas y terapéuticas no siempre congenian, porque sus lógicas son diferentes y las verdades resultantes quedan condicionadas muchas veces al objetivo de reconciliación entendido más como liberación del sufrimiento que como justicia objetiva, una justicia que por otra parte la Comisión no puede administrar porque no está incluida en su mandato. Por eso los objetivos de perdón y reconciliación resultaban controvertidos, Tutu los defendía como parte de un concepto de justicia restauradora más que retributiva por lo que iba más allá de una idea de justicia en el sentido jurídico de la palabra. 
MEMORIA, VERDAD, NACIÓN Y CIUDADANÍA: ALGUNAS

En lugar de violencia por violencia se ofrecía una cura mediante la posibilidad del perdón liberador. El concepto africano de Ubuntu fue promovido por el mismo Tutu como la noción legal y política clave para el orden post-apartheid inmediato. Ubuntu como expresión de una visión romántica de las comunidades rurales africanas, aludía a reciprocidad, respeto por la dignidad humana, cohesión de la comunidad y solidaridad (Wilson, 2001:9) y embonaba perfectamente con una idea de justicia basada en el entendimiento y no en la venganza, en la restauración y no en la represalia. En otras palabras, "justicia apropiada" versus venganza, justicia en pos del perdón y la reconciliación. El concepto aparece tanto en la Constitución interina como en la ley que dio origen a la comisión sentando las bases de la idea de justicia para la nueva cultura de derechos humanos que se quería establecer. Según Tutu se imponía así una idea africana de justicia (restauradora, que no busca tanto castigar como resarcir o restablecer el balance que ha sido torcido, quebrantado) en lugar de la idea retributiva de la justicia occidental (Minow, 1998: 81). Sin duda este razonamiento justificaba también las amnistías y ciertas faltas en el uso de sus poderes por parte de la comisión, como no realizar allanamientos en los cuarteles de las fuerzas armadas o en la sede del ANC o del Inkatha Freedom Party.

Entonces, a los requerimientos legales convencionales (a la manera de Chipoco) de "verdad factual objetiva" la TRC agregó: "verdad social o de diálogo" (a la que se llega mediante interacción, discusión y debate), "verdad narrativa" (percepciones, historias y mitos recitados por las víctimas) y "verdades de cura" (que colocaban el significado de los hechos dentro del contexto de las relaciones humanas).
¿Pero cuál es el pasado en cuestión? La ley de 1995 estableció que correspondía al período comprendido entre el 1 de marzo de 1960 y el 10 de Mayo de 1994. El término aplicable al período no puede ser otro que el de "apartheid" aunque, como señala Mark Sanders (2002: 63), fue una palabra reprimida en el discurso, reemplazada muchas veces por "conflictos del pasado" o "graves violaciones de los derechos humanos" y definida estrictamente como producto de un Estado que lo instrumentó mediante una serie de leyes de segregación y discriminación racial. De esta manera se restaba importancia a una interpretación del apartheid como una forma de explotación capitalista basada en la dominación etno-racial" o incluso se negaba la posibilidad de un análisis más profundo del mismo. Las víctimas del apartheid fueron individualizadas a pesar de que se reconoció al apartheid como "crimen contra la humanidad" que afectó a comunidades enteras con políticas de limpieza etnico-racial mediante clasificación y reubicación de la misma, todo acompañado de privación de derechos políticos y económicos. En este sentido, la comisión nunca incluyó la violación de derechos que implicaron las leyes de pases, los traslados forzosos de población, la discriminación en educación y oportunidades de empleo, etcétera.

\section{Conclusión}

Los testimonios y experiencias individuales a los que se dio voz son inscriptos en una narrativa correspondiente al marco institucional y discursivo de la TRC y en un contexto de compromiso político que la hizo posible pero a su vez estableció los limites de su accionar. Es decir que mediante toda una serie de definiciones como "víctimas" y "graves violaciones de los derechos humanos" ya en la ley 
que otorgó mandato a la TRC hasta el mismo proceso de tomar testimonio a las víctimas, la selección de los testimonios que debían ser presentados en las audiencias públicas, la selección de dichos testimonios por los medios para ser transmitidos a todo el país, etcétera, los testimonios han sido ajustados a una particular narrativa de la nueva historia de Sudáfrica. Las verdades resultantes, por supuesto parciales e incompletas, han sido sujetas a las exigencias de un espacio testimonial inscripto en un proyecto de cura, perdón y reconciliación que en el caso sudafricano ha tenido un componente psico-teológico-moral, y está enmarcado en un proyecto político de construcción-reconstrucción nacional. Narrativa intrínseca al discurso que construye una nueva identidad nacional, que inscribe un nuevo significado a la definición de "auténtico sudafricano": debe compartir los traumas del apartheid y unirse en el proceso de cura de la nación. El concepto de "Rainbow nation o rainbow people” (nación arcoiris) acuñado por el mismo Tutu fue el complemento para definir a la nueva nación como amalgama multicultural de distintos colores (unidos pero distintos). Y así, mientras otros países tienen sus días de rememoración de los héroes y mártires nacionales, libertadores de la nación, en Sudáfrica se instituyó el "día de la reconciliación", el 16 de diciembre día en que se reunió por primera vez el TRC, con el que se conmemora a un nuevo grupo de mártires nacionales que cayeron en manos de sus compatriotas sudafricanos.

Notas

1 Resultados parciales del proyecto Democracia, comunicación y sujetos de la politica, financiado por CONACYT, proyecto 42,715.

2 En 1993 el ANC estableció una comisión (la Comisión Motsuenyane) para investigar la violación de derechos humanos en algunos de sus campos en el exilio y el grado de trasgresión en sus normas de conducta. La Comisión corroboró la violación de derechos y nombró a los perpetradores. Se trata del primer caso en que un movimiento de liberación promueve una comisión independiente para investigar su propio pasado de abusos a los derechos humanos. Ver Motsuenyane Comission Report (23 de agosto de 1993); "Reponse of the NEC of the ANC to the Motsuenyane Commission Report" (28 de agosto de 1993) citado en Villa-Vicencio y Verwoerd (2000: 20).

${ }^{3}$ Decreto 187 del 15 de diciembre de 1983 y decreto 355 del 24 de abril de 1990, respectivamente.

${ }^{4}$ Acuerdo de México $(27$ de abril 1991) firmado entre el gobierno de El Salvador y el Frente Farabundo Martí para la Liberación Nacional, FMLN, con el auspicio de la ONU y Acuerdo de Oslo (23 de julio 1994) entre el Gobierno y la guerrilla, con el auspicio de la ONU.

${ }^{5}$ Además y con características diferentes en origen y contenido, en febrero de 2000, la Comisión de Relaciones Exteriores del parlamento belga estableció una comisión para investigar las circunstancias del asesinato de Patrice Lumumba en 1961 y las posibles implicaciones políticas de Bélgica.

${ }^{6}$ South Africa, Promotion 3[1][a].

${ }^{7}$ En Argentina, el mandato de la Comisión fue exclusivamente en relación con los detenidos desaparecidos y, por tanto, sólo para hechos perpetrados por el Estado (entre 1976 y 1983). En Chile, el mandato de la Comisión fue sólo para casos en que la víctima murió o permanecía a la fecha en situación de detenido desaparecido (casos acaecidos entre el 11 de septiembre de 1973 y el 11 de marzo de 1990). En El Salvador y Guatemala se tomó en consideración todo tipo de violación a los derechos fundamentales y el objetivo fundamental fue descubrir información sobre los desaparecidos.

${ }^{8}$ Ver el capítulo cuatro del Volumen uno del Reporte.

${ }^{9} \mathrm{La}$ Comisión argentina tuvo 13 comisionados, la chilena siete, Guatemala y El Salvador tres, respectivamente. ${ }^{10} \mathrm{La}$ de Argentina y la de Chile duraron nueve meses, respectivamente. En el caso de Chile, la Comisión recomendó el establecimiento de un nuevo organismo, el cual fue creado por ley. La nueva entidad se denominó "Corporación Nacional de Reparación 
MEMORIA, VERDAD, NACIÓN Y CIUDADANÍA: ALGUNAS

REFLEXIONES SOBRE LA COMISIÓN DE LA VERDAD

Y LA RECONCILIACIÓN EN SUdÁFRICA

y Reconciliación” y, de acuerdo con su mandato, procedió a otorgar la reparación económica a las víctimas declaradas por la Comisión de la Verdad, recibiendo nuevamente información durante doce meses (1992-1993) e incrementando así el número de víctimas reconocidas por el Estado. La de El Salvador ocho meses y la de Guatemala dieciocho meses.

${ }^{11}$ Metáfora también presente en algunas de las comisiones para América Latina; por ejemplo, para el caso de Uruguay señala Luis Pérez Aguirre: "Se ha dicho que hurgar en estos acontecimientos del pasado es abrir nuevamente las heridas del pasado. Nosotros nos preguntamos, por quién y cuándo se cerraron esas heridas. Ellas están abiertas y la única manera de cerrarlas será logrando una verdadera reconciliación nacional que se asiente sobre la verdad y la justicia respecto de lo sucedido. La reconciliación tiene esas mínimas y básicas condiciones. Luis Pérez Aguirre, "El Uruguay Impune y la memoria social". Tribunal Permanente de los Pueblos. Sesión Uruguay. Abril 1990. Montevideo, Uruguay: 31.

\section{Bibliografía}

Brahona de Brito, Alexandra, ed., 2001, Politics of Memory: Transitional Justice in Democratizing Societies, Oxford University Press.

Berryman, Phillip E., trans., 1993, Report of the Chilean National Commission on Truth and Reconciliation, University of Notre Dame Press.

Boraine, Alex, A, 2001, Country Unmasked: Inside South Africa's Truth and Reconciliation Commission, Oxford University Press.

Chipoco, Carlos, 1994, "El derecho a la verdad", en Paz, N 28. pp. 83 al 106, Lima, marzo.

Dyzenhais, David, 1998, Judging the Judges and Ourselves: Truth, Reconciliation and the Apartheid Legal Order, Northwestern University Press.

Edelstein, Jillian with Pumla Godbodo-Madikizela,2002, Truth and Lies: Stories from the Truth and Reconciliation Commission in South Africa, New Press.

Godwin Phelps, Teresa, 2004, Shattered Voides. Language, Violence, and the Work of Truth Commissions, Philadelphia, University of Pennsylvania Press.

Grinebaum-Ralph, Heidi, 2001, "Re-Placing Pasts, Forgetting Presents: Narrative, Place, and Memory in the Time of the Truth and Reconciliation Commission", Research in African Literatures Vol. 32, N.3, pp. 98-212.

Hayner, Priscilla B. 2002, Unspeakable Truths: Facing the Challenge of Truth Commissions, Taylor \& Francis, Inc.

-- ,1994, "Fifteen Truth Commissions-1974 to 1994: A Comparative Study”, Human Rights Quarterly, v. 16, no. 4, November, pp. 597-655

James, Wilmot and Linda Van de Vijver, 2001, After the TRC: Reflections on Truth and Reconciliation, (Ohio University Press.

Klopper, Dirk, 2001, "Narrative Time and the Space of the Image: The Truth of the Lie in Winnie Madikizela-Mandela's Testimony before the Truth and Reconciliation Commission”, Poetics Today 22:2, pp. 453-474.

Krog, Antjie, 2000, Country of My Skull: Guilt, Sorrow, and the Limits of Forgiveness in the New South Africa, Crown Publishing Group.

Minow, Martha, 1999, Between Vengeance and Forgiveness: Facing History After Genocide and Mass Violence, Beacon Press.

Reitzes, Maxine, 1995, "Insiders and Outsiders: The Reconstruction of Citizenship in South Africa", Policy:Issues and Actors, Vol. 8, n.1, Johannesburg, Centre for Policy Studies, Feb.

Sanders, Mark, 2001, "Remembering Apartheid", Diacritics 32.3-4:60-80.

The Official Truth and Reconciliation Website, TRC Report, 1999,http://www.doj.gov.za/trc_frameset.htm Truth and Reconciliation Commission of South Africa Report (London, Macmillan Reference Limited).

Tutu, Desmond Mpilo, 2000, No Future without Forgiveness (Doubleday \& Co., Inc).

VanZanten Gallagher, Susan, 1998, "Cry with a Beloved Country: Restoring Dignity to the Victims of Apartheid", Christianity Today, Feb. 9.

http://www.christianitytoday.com/ct/8t2/8t218a. html Villa-Vicencio, Charles and W. Verwoerd, 2000, Looking Back Reaching Forward. Reflections on the Truth and Reconciliation Commission of South Africa, Cape Town and London, University of Cape Town Press and Zed Books.

Wilson, Richard, 2001, Politics of Truth and Reconciliation in South Africa: Legitimizing the Post-Apartheid Stat, Cambridge University Press. 